\title{
EQUIVALENCE OF CONNECTIVITY MAPS AND PERIPHERALLY CONTINUOUS TRANSFORMATIONS
}

\author{
MELVIN R. HAGAN
}

In [1] and [2] O. H. Hamilton and J. Stallings have shown that a local connectivity mapping, and hence a connectivity mapping, of a locally peripherally connected polyhedron into a regular Hausdorff space is peripherally continuous. The purpose of this paper is to prove the converse of this theorem.

Some definitions will now be recalled. A mapping $f: S \rightarrow T$ is a connectivity mapping if for every connected set $A$ in $S$, the set $g(A)$ is connected, where $g: S \rightarrow S \times T$ is the graph map of $f$ defined by $g(p)=(p, f(p))[1$, p. 750]. The mapping $f$ is a local connectivity mapping if there is an open covering $\left\{U_{\alpha}\right\}$ of $S$ such that $f \mid U_{\alpha}$ is a connectivity mapping for every $\alpha$ [2, p. 249]. The mapping $f$ is peripherally continuous if for every point $p$ in $S$ and for every pair of open sets $U$ and $V$ containing $p$ and $f(p)$, respectively, there is an open set $N \subset U$ and containing $p$ such that $f(F(N)) \subset V$, where $F(N)$ is the boundary of $N[1, \mathrm{p} .751]$. A space $S$ is locally peripherally connected if every point has arbitrarily small neighborhoods with connected boundary [2, p. 252].

In this paper $S$ will denote a connected, locally connected, locally peripherally connected, unicoherent metric space and $T$ a space such that $S \times T$ is completely normal.

The following lemma, proved by Stallings [2, p. 255], is used in the proof of Theorem 1.

Lemma 1. If $f: S \rightarrow T$ is peripherally continuous, then for every point $p$ in $S$ and every pair of open sets $U$ and $V$ containing $p$ and $(p, f(p))$, respectively, there is an open connected set $N \subset U$ and containing $p$ such that $F(N)$ is connected and $g(F(N)) \subset V$.

Lemma 2. Let $W$ be an open connected subset of $S$ such that $F(W)$ is connected. Let $W_{1}$ and $W_{2}$ be open connected sets such that $W_{1} \cap W_{2} \neq \varnothing$, $F\left(W_{1}\right)$ and $F\left(W_{2}\right)$ are connected, and $\mathrm{cl}\left(W_{1}\right) \cup \mathrm{cl}\left(W_{2}\right) \subset W$. Then there is a connected open set $W_{3}$ such that (1) $W_{1} \cup W_{2} \subset W_{3} \subset W$, (2) $F\left(W_{3}\right)$ is contained in $F\left(W_{1}\right) \cup F\left(W_{2}\right)$, and (3) $F\left(W_{3}\right)$ is connected.

Proof. The proof is similar to the proof of Lemma 1. Let $X$ $=W_{1} \cup W_{2}$. Then $F(X)$ is connected and separates $F(W)$ and $X$. Let

Received by the editors July 6, 1964. 
$C=F(X) \cup\{y \in W ; F(X)$ separates $y$ and $F(W)\}$ and $W_{3}=$ component of int $C$ containing $X$. Then by standard theorems concerning unicoherence [3, p. 51], $F\left(W_{3}\right) \subset F(X)$ and $F\left(W_{3}\right)$ is connected.

The following theorem is the converse of Hamilton's and Stallings' theorem.

THEOREM 1. If $f: S \rightarrow T$ is peripherally continuous, then $f$ is a connectivity map.

Proof. Suppose that $f$ is not a connectivity map and let $A$ be a connected subset of $S$ such that $g(A)=M \cup N$, where $M$ and $N$ are separated. Let $g^{-1}(M)=H$ and $g^{-1}(N)=K$. Then $A=H \cup K$, where $H \cap K=\varnothing$. Since $A$ is connected $H$ and $K$ are not separated and hence one must contain a limit point of the other. Let $p$ be a point of $H$ that is a limit point of $K$. Since $S \times T$ is completely normal there exist open disjoint sets $U$ and $V$ in $S \times T$ containing $M$ and $N$, respectively.

Let $R$ be an open set containing $p$ such that $A$ is not contained entirely in $R$. By Lemma 1 there is an open connected set $W$ containing $p$ and contained in $R$ such that $W$ and $F(W)$ are both connected and $g(F(W)) \subset U$. Since $p$ is a limit point of $K$ there is a point $q$ of $K$ in $W$.

Let $Q$ be the collection of all open connected sets $D$ such that $q$ is in $D, \operatorname{cl}(D) \subset W, F(D)$ is connected, and $g(F(D)) \subset V$. The collection $Q$ is nonempty since $f$ is peripherally continuous at the point $q$. Denote by $Q^{*}$ the point-set union of all sets in $Q$. Then $Q^{*}$ is an open subset of $W$. Since the connected set $A$ intersects both $Q^{*}$ and $S-Q^{*}$, it follows that $A \cap F\left(Q^{*}\right) \neq \varnothing$.

Since $F\left(Q^{*}\right) \cap A \neq \varnothing$, then $F\left(Q^{*}\right)$ either contains a point of $H$ or a point of $K$. Suppose there is a point $h$ in $F\left(Q^{*}\right) \cap H$. Then there is an open set $E$ containing $h$ but not $q$ such that $F(E)$ is connected and $g(F(E)) \subset U$. Since $h$ is a limit point of $Q^{*}, E$ must intersect some set $D$ belonging to the collection $Q$. Now $E \nsubseteq D$ since $h$ is in $E-D$ and $D \subset E$ since $q$ is in $D-E$. Thus $E$ and $D$ both have points interior and exterior to one another and $F(D)$ and $F(E)$ being connected implies $F(D) \cap F(E) \neq \varnothing$. But this contradicts the fact that $g(F(D))$ $\subset V, g(F(E)) \subset U$ and $U \cap V=\varnothing$. Hence $F\left(Q^{*}\right) \cap H=\varnothing$ and therefore $F\left(Q^{*}\right) \cap K \neq \varnothing$.

Let $k$ be a point of $F\left(Q^{*}\right) \cap K$. Now $k$ is not a point of $F(W)$ since $g(F(W)) \subset U$ and $g(k)$ is in $V$. Thus $k$ is in $W$ and there is an open connected set $W_{1}$ containing $k$ and contained in $W$ such that $F\left(W_{1}\right)$ is connected, $\operatorname{cl}\left(W_{1}\right) \subset W$ and $g\left(F\left(W_{1}\right)\right) \subset V$. Since $k$ is a limit point of $Q^{*}$ there is a set $W_{2}$ in the collection $Q$ such that $W_{1} \cap W_{2} \neq \varnothing$. 
Now form the set $W_{3}$ referred to in Lemma 2. By this lemma the set $W_{3}$ is open, connected, $F\left(W_{3}\right)$ is connected, $\operatorname{cl}\left(W_{3}\right) \subset W$, and $q$ is in $W_{3}$. Further, $g\left(F\left(W_{3}\right)\right) \subset V$ since $F\left(W_{3}\right) \subset F\left(W_{1}\right) \cup F\left(W_{2}\right)$. Therefore $W_{3}$ possesses all the requirements to belong to $Q$, but $W_{3}$ is not in $Q$ since $k$ is in $\left(W_{3} \cap F\left(Q^{*}\right)\right)$. Therefore the assumption that $g(A)$ is not connected leads to a contradiction. Hence $f$ is a connectivity map.

Stallings' theorem, [2, p. 253], and Theorem 1 imply, in particular, that on an $n$-cell, $n \geqq 2$, into itself there is no distinction among local connectivity maps, connectivity maps, and peripherally continuous transformations. Thus, the question posed on p. 752 of [1] and question 5, p. 262 of [2] are answered. The following theorem will complete the theory of equivalence of the local connectivity maps and the connectivity maps of an $n$-cell, $n=1,2, \cdots$, in to itself.

THEOREM 2. If $f$ is a local connectivity map of the closed unit interval $I$ into itself, then $f$ is a connectivity map.

Proof. Since $f$ is a local connectivity map there is an open covering $\left\{U_{\alpha}\right\}$ of $I$ such that $f$ restricted to $U_{\alpha}$ is a connectivity map for each $\alpha$. Since $I$ is compact the covering $\left\{U_{\alpha}\right\}$ can be reduced to an irreducible number of intervals $I_{1}, \cdots, I_{n}$, such that $I_{i} \cap I_{i+1} \neq \varnothing$, and $f$ is a connectivity map on each $I_{i}$. Then if $K$ is any connected subset of $I, K$ is an interval and $K=\left(K \cap I_{1}\right) \cup \ldots \cup\left(K \cap I_{n}\right)$, where each $K \cap I_{i}$ is an interval contained in $I_{i}$. Thus $g\left(K \cap I_{i}\right)$ is connected and since $g\left(K \cap I_{i}\right) \cap g\left(K \cap I_{i+1}\right) \neq \varnothing, g(K)$ is connected. Therefore $f$ is a connectivity map.

\section{REFERENCES}

1. O. H. Hamilton, Fixed points for certain noncontinuous transformations, Proc. Amer. Math. Soc. 8 (1957), 750-756.

2. J. Stallings, Fixed point theorems for connectivity maps, Fund. Math. 48 (1959), 249-263.

3. R. L. Wilder, Topology of manifolds, Amer. Math. Soc. Colloq. Publ. Vol. 32, Amer. Math. Soc., Providence, R. I., 1949.

OkIahoma State University 